\title{
Testing Hypotheses About Methods, Traits, and Communalities in the Direct-Product Model
}

\author{
Richard P. Bagozzi and Youjae Yi \\ University of Michigan
}

The direct-product model has been suggested as a procedure for estimating multiplicative effects of traits and methods in multitrait-multimethod matrices. Research on the direct-product model is extended in two ways. First, hierarchically nested models are derived for explicitly testing the overall and specific patterns of method and trait factors. Second, formal tests are developed for the pattern of communalities. These procedures are illustrated with data from Lawler (1967). Index terms: directproduct model, method factors, multiplicative model, multitrait-multimethod matrix, trait factors.

Method factors may interact with trait factors multiplicatively in the multitrait-multimethod (MTMM) matrix (e.g., Campbell \& O'Connell, 1967, 1982; Schmitt \& Stults, 1986). Building on a model proposed by Swain (1975), Browne (1984) formally represented the interaction of traits and methods in a direct-product model (DPM) and developed the program MUTMUM, which estimates parameters and tests the general model (Browne, 1991). The DPM can be reformulated as a linear model (Wothke \& Browne, 1990); therefore, the DPM can be fitted with programs such as EQS (Bentler, 1989; Bentler, Poon, \& Lee, 1988) and LISREL (Jöreskog \& Sörbom, 1989).

Given the frequent use of MTMM designs and the availability of these programs, it would seem likely that many researchers would employ the DPM in construct validation studies. However, the DPM has been used infrequently by applied researchers-very few studies could be found in the psychology literature (e.g., Bagozzi \& Yi,

APPLIED PSYCHOLOGICAL MEASUREMENT

Vol. 16, No. 4, December 1992, pp. 373-380

(C) Copyright 1992 Applied Psychological Measurement Inc. 0146-6216/92/040373-08\$1.65
1990; Cudeck, 1988). Undoubtedly, the relative newness of the model accounts for its infrequent application to date. Further, the procedures for analyzing and interpreting the DPM are not straightforward for many researchers performing substantive investigations.

Although many contributions have been made to the theoretical formulation and estimation of the DPM in the psychometric literature, relatively little attention has been given to its practical application. Consequently, the literature provides little guidance on how to use the DPM in analyzing MTMM data. For example, applications to date have focused only on the appropriateness and fit of the overall model (e.g., Bagozzi, 1991; Bagozzi \& Yi, 1990, 1991; Bagozzi, Yi, \& Phillips, 1991; Cudeck, 1988; Lastovicka, Murry, \& Joachimsthaler, 1990). More detailed hypotheses about traits and methods need to be specified, and formal tests of hypotheses of construct validity should be developed.

The present paper develops procedures for specifying and testing meaningful hypotheses regarding construct validity when traits and methods interact. Specifically, two extensions in the use of DPMs are presented. First, a series of hierarchically nested models for testing hypotheses of specific trait and method patterns are derived. This approach is analogous to Widaman's (1985) taxonomy of structural models for the linear formulation (i.e., confirmatory factor analysis), but because of the nature of the multiplicative interaction between traits and methods, the derivation and interpretation of the DPM are not straightforward. Second, formal tests of some meaningful hypotheses about 
communalities are developed. These procedures are illustrated with data from Lawler (1967), which previously has been used to introduce researchers to the DPM (e.g., Wothke \& Browne, 1990).

\section{The Direct-Product Model}

Swain (1975) proposed the DPM to represent the multiplicative interaction of traits and methods in the MTMM matrix:

$\Sigma=\Sigma_{\mathrm{M}} \otimes \Sigma_{\mathrm{T}}$,

where $\Sigma$ is the covariance matrix of the observed variables;

$\Sigma_{\mathrm{M}}$ and $\Sigma_{\mathrm{T}}$ are method and trait covariance matrices, respectively; and

$\otimes$ indicates a right direct (Kronecker) product.

However, because this model does not allow for measurement errors or for different scales for different variables, the applicability of the model is limited in many MTMM studies. Browne (1984, 1989) extended the DPM to overcome these limitations (see also Cudeck, 1988):

$\mathbf{\Sigma}=\mathbf{Z}\left(\mathbf{P}_{\mathrm{M}} \otimes \mathbf{P}_{\mathrm{T}}+\mathbf{E}\right) \mathbf{Z}$,

where $\mathbb{Z}$ is a non-negative definite diagonal matrix of scale constants;

$\mathbf{P}_{\mathrm{M}}$ and $\mathbf{P}_{\mathrm{T}}$ are non-negative definite method and trait correlation matrices, respectively; and

$\mathbf{E}$ is a diagonal matrix of non-negative unique variances.

This model, which is called a heteroscedastic error model, allows for measurement error for different variables (Wothke \& Browne, 1990). Note that Equation 2 decomposes test scores into common factor score and error score components. Thus, the common factor correlation (corrected for attenuation) has a direct-product structure,

$\mathbf{P}_{\mathrm{C}}=\mathbf{P}_{\mathrm{M}} \otimes \mathbf{P}_{\mathrm{T}}$.

One useful version of the DPM is defined by adding a restriction (i.e., $\mathbf{E}=\mathbf{E}_{\mathrm{M}} \otimes \mathbf{E}_{\mathrm{T}}$, with $\mathbf{E}_{\mathrm{M}}$ and $\mathbf{E}_{\mathrm{T}}$ diagonal) that yields
$\mathbf{\Sigma}=\mathbb{Z}\left(\mathbf{P}_{\mathrm{M}} \otimes \mathbf{P}_{\mathrm{T}}+\mathbf{E}_{\mathrm{M}} \otimes \mathbf{E}_{\mathrm{T}}\right) \mathbf{Z}$

This is called a composite error model (Wothke $\&$ Browne, 1990). Thus, there are two versions of the DPM: the more general heteroscedastic error model given in Equation 2, and a composite error model given in Equation 4. The program MUTMUM (Browne, 1991) estimates the parameters in the DPM.

\section{Testing Hypotheses for Methods and Traits}

To introduce a set of procedures for formally testing hypotheses concerning the effects of traits and methods, it is useful to consider the simple case with three traits and three methods. (These procedures are applicable to other MTMM matrices.) Suppose the correlation matrices among methods and among traits can be written, respectively, as

$\mathbf{P}_{\mathrm{M}}=\left[\begin{array}{ccc}1 & & \\ \rho_{\mathrm{m} 21} & 1 & \\ \rho_{\mathrm{m} 31} & \rho_{\mathrm{m} 32} & 1\end{array}\right]$,

and

$\mathbf{P}_{\mathrm{T}}=\left[\begin{array}{ccc}1 & & \\ \rho_{\mathrm{t} 21} & 1 & \\ \rho_{\mathrm{t} 31} & \rho_{\mathrm{t} 32} & 1\end{array}\right]$.

Five useful hypotheses about methods are:

$\mathrm{H}_{1}$ : Method correlations follow a Toeplitz pattern;

$\mathrm{H}_{2}$ : All methods are equally correlated;

$\mathrm{H}_{3}$ : All methods are completely unrelated;

$\mathrm{H}_{4}$ : All methods are equivalent; and

$\mathrm{H}_{5}$ : Some methods are equivalent.

$\mathrm{H}_{1}$ is especially suitable for multitest-multioccasion data in which methods represent occasions. It posits that the method correlation matrix is a Toeplitz matrix of the form

$\mathbf{P}_{M}=\left[\begin{array}{llll}1 & & & \\ \rho_{1} & 1 & & \\ \rho_{2} & \rho_{1} & 1 & \\ \rho_{3} & \rho_{2} & \rho_{1} & 1\end{array}\right]$. 
If the occasions are equally spaced, the correlation between occasions (i.e., methods) depends on the interval between them. In applications to typical multitest-multioccasion data, $\rho_{1} \geq \rho_{2} \geq \rho_{3}$ would be obtained. For the MTMM data with three methods, $\mathrm{H}_{1}$ yields the restriction $\rho_{\mathrm{m} 21}=\rho_{\mathrm{m} 32}$.

$\mathrm{H}_{2}$ posits that all methods are equally correlated. $\mathrm{H}_{2}$ implies that all nondiagonal elements of $\mathbf{P}_{\mathrm{M}}$ are equal. Thus, $\mathrm{H}_{2}$ yields the restriction $\rho_{m 21}=\rho_{m 31}=\rho_{m 32}(=\rho)$. According to $\mathrm{H}_{2}$, whether a method is shared or not influences heterotrait correlations. For example, the common factor correlations involving Traits 1 and 2 would be $\rho_{t 21}$ within each method, whereas they are $\rho_{\mathrm{m} 21} \rho_{\mathrm{t} 21}=\rho_{\mathrm{m} 31} \rho_{\mathrm{t} 21}=\rho_{\mathrm{m} 32} \rho_{\mathrm{t} 21}=$ $\rho p_{t 21}$ across different methods (see Equation 3). That is, once different methods are used, any combination of methods leads to the same effect.

$\mathrm{H}_{3}$ suggests that all methods are orthogonal or that all nondiagonal elements of $\mathbb{P}_{M}$ are 0 . That is, $\mathrm{H}_{3}$ implies the restriction $\rho_{\mathrm{m} 21}=$ $\rho_{\mathrm{m} 31}=\rho_{\mathrm{m} 32}=0.0 . \mathrm{H}_{4}$ posits that all methods are equivalent (i.e., $H_{4}: M_{1}=M_{2}=M_{3}$ ). $H_{4}$ implies the restriction that all methods are perfectly correlated: $\rho_{\mathrm{m} 21}=\rho_{\mathrm{m} 31}=\rho_{\mathrm{m} 32}=1.0$. Thus, $\mathrm{H}_{4}$ represents complete redundancy in methods; that is, a single method factor is sufficient to represent method effects. A formal test can be conducted by comparing the full DPM with the restriction $\rho_{\mathrm{n} 21}=\rho_{\mathrm{m} 31}=\rho_{\mathrm{m} 32}=1.0$. Note that $\mathrm{H}_{3}$ and $\mathrm{H}_{4}$ are nested versions of $\mathrm{H}_{2}$.

When $\mathrm{H}_{4}$ is rejected, it is still possible that one or more pairs of methods might not be equivalent $\left(\mathrm{H}_{5}\right)$. For example, a researcher could investigate whether any methods used in a pretest are redundant in order to avoid unnecessary duplication in subsequent research, so that the length of a survey as well as the potential boredom effect among respondents could be reduced. Thus, whether a particular pair of methods is equivalent could be tested. For instance, it may be hypothesized that Methods 1 and 2 are equivalent (i.e., $M_{1}=M_{2}$ ). This requires the restriction that the two methods be perfectly correlated (i.e., $\rho_{\mathrm{m} 21}=1.0$ ). However, this restriction implies another restriction-both methods must be equally correlated with the other methods (i.e., $\rho_{\mathrm{m} 2 r}=\rho_{\mathrm{m} 1 r}, r \neq 1$, 2); otherwise, $\mathbf{P}_{\mathrm{M}}$ will not be positive definite or semidefinite (Browne, 1991). Thus, the hypothesis of equivalence between Methods 1 and $2\left(M_{1}=M_{2}\right)$ can be tested with the following restrictions: $\rho_{\mathrm{m} 21}=1.0$ and $\rho_{\mathrm{m} 23}=$ $\rho_{\mathrm{m} 13}$.

Four useful hypotheses regarding traits are:

$\mathrm{H}_{6:}$ All traits are equally correlated;

$\mathrm{H}_{7}$ : All traits are completely unrelated;

$\mathrm{H}_{8}$ : All traits are equivalent; and

$\mathrm{H}_{9}$ : Some traits are equivalent.

$\mathrm{H}_{6}$ posits that all traits are equally correlated. This hypothesis implies that all trait correlations are the same; that is, $\rho_{112}=\rho_{t 13}=\rho_{t 23}(=\rho)$. Thus, whether a common trait is involved or not affects heteromethod correlations. For example, the correlations involving Methods 1 and 3 under each trait would be $\rho_{\mathrm{m} 31}$, whereas those across different traits are $\rho_{\mathrm{t} 21} \rho_{\mathrm{m} 31}=$ $\rho_{131} \rho_{\mathrm{m} 31}=\rho_{\mathrm{t32}} \rho_{\mathrm{m} 31}=\rho \rho_{\mathrm{m} 31}$. That is, any combination of traits leads to the same effect when different traits are involved.

$\mathrm{H}_{7}$ says that all traits are completely orthogonal. Thus, this hypothesis yields the restriction $\rho_{121}=\rho_{131}=\rho_{132}=0.0 . H_{8}$ posits that all traits are equivalent. This hypothesis suggests that all traits are the same (i.e., $T_{1}=T_{2}=T_{3}$ ), or that traits do not make a difference in the heteromethod correlations.

When $\mathrm{H}_{8}$ is rejected, it is meaningful to test whether some traits are equivalent $\left(\mathrm{H}_{9}\right)$. For instance, it may be hypothesized that Traits 2 and 3 are equivalent (i.e., $T_{2}=T_{3}$ ). This hypothesis requires the restriction that the correlation between the two traits be unity (i.e., $\rho_{123}=1.0$ ). Again, this restriction implies another restriction that both traits be equally correlated with the other traits (i.e., $\rho_{\mathrm{t} 2 r}=\rho_{\mathrm{t} 3 r}, r \neq 2,3$ ). Thus, the hypothesis of equivalence between Traits 2 and $3\left(\mathrm{~T}_{2}=\mathrm{T}_{3}\right)$ can be tested with the restrictions $\rho_{132}=1.0$ and $\rho_{131}=\rho_{121}$. 


\section{An Illustration}

Method. Lawler's (1967) data were used to illustrate these hypotheses. The MTMM matrix is given in Table 1. Three traits are represented: quality of job performance, ability to perform on the job, and effort put forth on the job. The traits were measured with three methods: superior ratings, peer ratings, and self-ratings.

MUTMUM (Browne, 1991) was used to investigate DPMs. Although LISREL or EQS could be used by following Wothke \& Browne's (1990) parameterization of the DPM as a linear model, MUTMUM was selected for the following reasons. MUTMUM estimates the trait and method correlation matrices simultaneously and provides standard errors for both trait and method correlations. In contrast, a particular LISREL or EQS run only computes standard errors for trait or method correlations; the model then must be reparameterized and the program run again to yield both estimates. Also, MUTMUM accommodates equality constraints simultaneously on both trait and method correlation matrices; this cannot be done with LISREL or EQS under the DPM. Furthermore, LISREL sometimes results in empirical underidentification, but MUTMUM is not as sensitive to such occurrences (e.g., Bagozzi \& Yi, in press). MUTMUM also automatically imposes inequality constraints for some parameter estimates; for example, trait and method correlations are bounded between -1 and +1 (Browne, 1991, p. 4). These inequality constraints result in maximum likelihood estimates in some circumstances in which the likelihood function would be unbounded otherwise.

Results. The full DPM specified in Equation 4 first was estimated (see Model A in Table 2). The model fit was satisfactory (this model also was estimated with LISREL following the procedure by Wothke \& Browne, 1990, and the results were the same as those obtained by the MUTMUM analysis). Next, a series of restricted DPMS were fit to investigate the pattern of individual method factors. $\chi^{2}$ tests then were used to test the overall hypotheses as well as specific hypotheses about differences between methods (see Table 2).

$\mathrm{H}_{1}$ was tested by comparing the full model (Model A) and the restricted model with a Toeplitz pattern (Model B). The $\chi^{2}$ difference test (test of Model A vs. Model B in Table 2) indicated that $\mathrm{H}_{1}$ could be rejected. $\mathrm{H}_{2}$ posited that all method correlations were equal (Model C). The $\chi^{2}$ difference test (Model A vs. Model $\mathrm{C}$ ) was significant at the .001 level. Thus, $\mathrm{H}_{2}$ could be rejected. $\mathrm{H}_{3}$, which posited that all methods were completely unrelated, was tested by comparing Models A vs. $\mathrm{D}$; the $\chi^{2}$ difference was significant at the .001 level, suggesting that $\mathrm{H}_{3}$ be rejected. $\mathrm{H}_{4}$ is an omnibus hypothesis that all methods

Table 1

Lawler's (1967) Managerial Job Performance Data $(N=113)$

[Copyright (1967) American Psychological Association. Reproduced by Permission.]

\begin{tabular}{lrrrrrrrrr}
\hline \hline Rater and Variable & Su-Q & Su-A & Su-E & P-Q & P-A & P-E & Se-A & Se-A & Se-E \\
\hline Superior (Su) & & & & & & & & & \\
Quality (Q) & 1.00 & & & & & & & & \\
Ability (A) & .53 & 1.00 & & & & & & & \\
Effort (E) & .56 & .44 & 1.00 & & & & & & \\
Peer (P) & & & & & & & & & \\
$\quad$ Quality (Q) & .65 & .38 & .40 & 1.00 & & & & & \\
Ability (A) & .42 & .52 & .30 & .56 & 1.00 & & & & \\
Effort (E) & .40 & .31 & .53 & .56 & .40 & 1.00 & & & \\
Self (Se) & & & & & & & & \\
Quality (Q) & .01 & .01 & .09 & .01 & .17 & .10 & 1.00 & & \\
Ability (A) & .03 & .13 & .03 & .04 & .09 & .02 & .43 & 1.00 & \\
$\quad$ Effort (E) & .06 & .01 & .30 & .02 & .01 & .30 & .40 & .14 & 1.00 \\
\hline
\end{tabular}


Table 2

Results of $\chi^{2}$ Goodness-of-Fit Tests for Models and $\chi^{2}$ Difference Tests for Hypotheses About Methods, Traits, and Communalities

\begin{tabular}{|c|c|c|c|}
\hline Model and Test & $d f$ & $\chi^{2}$ & $\begin{array}{c}\text { Approximate } \\
p \text { value }\end{array}$ \\
\hline \multicolumn{4}{|l|}{ Method Model } \\
\hline A. Trait $\times$ Method Model & 25 & 28.94 & .27 \\
\hline B. Toeplitz-Pattern Model & 26 & 54.56 & $<.001$ \\
\hline C. Equally Correlated Methods & 27 & 59.55 & $<.001$ \\
\hline D. Completely Unrelated Methods & $29^{a}$ & 137.38 & $<.001$ \\
\hline E. All Equivalent Methods & 30 & 185.39 & $<.001$ \\
\hline F. Methods 1 \& 2 Equivalent & 28 & 65.14 & $<.001$ \\
\hline G. Methods $2 \& 3$ Equivalent & 28 & 150.27 & $<.001$ \\
\hline H. Methods 1 \& 3 Equivalent & 28 & 148.80 & $<.001$ \\
\hline \multicolumn{4}{|l|}{ Test of } \\
\hline \multicolumn{4}{|l|}{ Toeplitz-Pattern Method } \\
\hline Correlations (A vs. B) & 1 & 25.62 & $<.001$ \\
\hline Equal Method Correlations (A vs. C) & 2 & 30.61 & $<.001$ \\
\hline Unrelated Methods (A vs. D) & 4 & 108.44 & $<.001$ \\
\hline $\mathrm{M}_{1}=\mathrm{M}_{2}=\mathrm{M}_{3}(\mathrm{~A}$ vs. $\mathrm{E})$ & 5 & 156.46 & $<.001$ \\
\hline $\mathrm{M}_{1}=\mathrm{M}_{2}(\mathrm{~A}$ vs. $\mathrm{F})$ & 3 & 36.20 & $<.001$ \\
\hline $\mathrm{M}_{1}=\mathrm{M}_{3}$ (A vs. $\left.\mathrm{G}\right)$ & 3 & 121.33 & $<.001$ \\
\hline $\mathrm{M}_{2}=\mathrm{M}_{3}(\mathrm{~A}$ vs. $\mathrm{H})$ & 3 & 119.86 & $<.001$ \\
\hline \multicolumn{4}{|l|}{ Trait Model } \\
\hline I. Trait $\times$ Method Model & 25 & 28.94 & .27 \\
\hline J. Equally Correlated Traits & 27 & 34.14 & .16 \\
\hline K. Completely Unrelated Traits & $30^{2}$ & 201.35 & $<.001$ \\
\hline L. All Equivalent Traits & 30 & 92.02 & $<.001$ \\
\hline M. Traits 1 \& 2 Equivalent & 28 & 66.11 & $<.001$ \\
\hline N. Traits 2 \& 3 Equivalent & 28 & 60.16 & $<.001$ \\
\hline O. Traits 1 \& 3 Equivalent & 28 & 71.73 & $<.001$ \\
\hline \multicolumn{4}{|l|}{ Test of } \\
\hline Equal Trait Correlations (I vs. J) & 2 & 5.20 & $>.05$ \\
\hline Unrelated Traits (I vs. $\mathbb{K}$ ) & 5 & 172.41 & $<.001$ \\
\hline $\mathrm{T}_{1}=\mathrm{T}_{2}=\mathrm{T}_{3}($ I vs. $\mathrm{L})$ & 5 & 63.08 & $<.001$ \\
\hline $\mathrm{T}_{1}=\mathrm{T}_{2}(\mathrm{I}$ vs. $\mathrm{M})$ & 3 & 37.17 & $<.001$ \\
\hline $\mathrm{T}_{1}=\mathrm{T}_{3}(\mathrm{I}$ vs. $\mathrm{N})$ & 3 & 31.22 & $<.001$ \\
\hline $\mathrm{T}_{2}=\mathrm{T}_{3}$ (I vs. $\left.\mathrm{O}\right)$ & 3 & 42.79 & $<.001$ \\
\hline \multicolumn{4}{|l|}{ Communality Model } \\
\hline P. Unrestricted $\mathbf{E}$ & 21 & 28.83 & .12 \\
\hline Q. $\mathbf{E}=\mathbf{E}_{\mathrm{M}} \otimes \mathbf{E}_{\mathrm{T}}$ & 25 & 28.94 & .27 \\
\hline R. $\overline{\mathbf{E}}_{\mathrm{M}}=\mathbb{I}$ & 27 & 31.88 & .24 \\
\hline S. $\mathbf{E}_{\mathrm{T}}=\mathbf{I}$ & 27 & 32.29 & .21 \\
\hline T. Equal diagonal elements of $\mathbb{E}$ & 29 & 34.53 & .22 \\
\hline \multicolumn{4}{|l|}{ Test of } \\
\hline $\mathbf{E}=\mathbf{E}_{\mathrm{M}} \otimes \mathbf{E}_{\mathrm{T}}(\mathrm{P}$ vs. $\mathrm{Q})$ & 4 & .11 & $>.99$ \\
\hline $\mathbf{E}=\mathbb{I} \otimes \mathbb{E}_{\mathrm{T}}(\mathrm{Q}$ vs. $\mathrm{R})$ & 2 & 2.94 & $>.20$ \\
\hline $\mathbf{E}=\mathbf{E}_{\mathrm{M}} \otimes \mathbb{I}(\mathrm{Q}$ vs. $\mathrm{S})$ & 2 & 3.35 & $>.10$ \\
\hline Equal diagonal elements of $\mathbb{E}$ (Q. vs. T) & 4 & 5.59 & $>.25$ \\
\hline
\end{tabular}

a The degrees of freedom were modified because the MUTMUM program automatically imposes inequality constraints on parameter estimates (Browne, 1991, p. 4). 
are equivalent; the $\chi^{2}$ difference test (A vs. E) suggested that $\mathrm{H}_{4}$ be rejected.

$\mathrm{H}_{5}$ posited that some of the methods might be equivalent. Tests of individual methods also were conducted by comparing the full trait $\times$ method model with restricted models. The results for the model with the equivalence constraint for Methods 1 and 2 (Model F) are provided in Table 2 . The results indicated that the hypothesis of equivalent Methods 1 and 2 should be rejected (Model A vs. Model F). The hypotheses of equivalence for Methods 2 and 3 (Model G) and equivalent Methods 1 and 3 (Model H) also were tested. Both hypotheses were rejected (see tests of Model A vs. Model G and Model A vs. Model H in Table 2).

Table 2 also includes the results for testing hypotheses about traits. As for methods, the trait $\times$ method model $\chi^{2}$ is a general goodnessof-fit index for the DPM. A series of restricted DPMs were fit to test hypotheses about trait patterns. $\chi^{2}$ difference tests then were used to test the significance of overall as well as individual patterns of traits.

$\mathrm{H}_{6}$ was tested by comparing the full DPM (Model I) and the restricted model with all equal trait correlations (Model J). The $\chi^{2}$ difference test (I vs. J) was not significant at the .05 level. $\mathrm{H}_{7}$ posited that all traits were completely unrelated. The $\chi^{2}$ difference test (Model I vs. K) was significant at the .001 level. $\mathrm{H}_{8}$ is an omnibus hypothesis that all traits are equivalent. The $\chi^{2}$ difference test (Model I vs. L) suggested that $\mathrm{H}_{8}$ be rejected. $\mathrm{H}_{9}$ tested the possibility that some traits might be equivalent (Models $\mathrm{M}, \mathrm{N}$, and $O$ ). The $\chi^{2}$ difference test results of Model $I$ vs. $M$ indicated that the hypothesis of equivalent Traits 1 and 2 (Model M) should be rejected. Other hypotheses were tested similarly, but neither Traits 2 and 3 (Model I vs. N) nor Traits 1 and 3 (Model I vs. O) were found to be equivalent.

\section{Testing Hypotheses for Communalities}

Hypotheses about the error matrix $\mathbb{E}$ also may be considered, because the patterns of $\mathbf{E}$ gen- erate corresponding patterns for communalities. Some useful hypotheses about errors are:

$$
\begin{aligned}
& \mathrm{H}_{10}: \mathbf{E} \text { is unrestricted; } \\
& \mathrm{H}_{11}: \mathbf{E}=\mathbf{E}_{\mathrm{M}} \otimes \mathbf{E}_{\mathrm{T}} ; \\
& \mathrm{H}_{12}: \mathbf{E}=\mathbf{I} \otimes \mathbf{E}_{\mathrm{T}} ; \\
& \mathrm{H}_{13}: \mathbf{E}=\mathbf{E}_{\mathrm{M}} \otimes \mathbb{I} ; \text { and } \\
& \mathrm{H}_{14}: \text { All diagonal elements of } \mathbf{E} \text { are the same; }
\end{aligned}
$$

where $I$ is the identity matrix. $\mathrm{H}_{10}$ implies a basic DPM, as in Equation 2, and imposes no structure on $\mathbb{E}$. That is, $\mathbb{E}$ is not restricted under $\mathrm{H}_{10}$. $\mathrm{H}_{11}$ defines a useful version of the DPM by adding the restriction $\mathbb{E}=\mathbb{E}_{\mathrm{M}} \otimes \mathbf{E}_{\mathrm{T}}$. This restriction implies that trait communalities have the same rank order within each method and that method communalities have the same rank order within each trait (Browne, 1991).

$\mathrm{H}_{12}$ causes the communality of each trait to be equal across methods. This hypothesis can be tested through the restricted model by fixing the diagonal matrix of errors corresponding to methods to unity; that is, $\mathbb{E}_{\mathrm{M}}=\mathbb{I}$ (see the expression in Equation 4). Similarly, $\mathrm{H}_{13}$ implies that the communality of each method is equal across traits. Thus, this hypothesis yields the restriction that $\mathbf{E}_{\mathrm{T}}$ is an identity matrix.

$\mathrm{H}_{14}$ posits that all diagonal elements of $\mathbb{E}$ are the same. This hypothesis implies that trait communalities remain the same under all methods and that method communalities remain the same under all traits.

\section{An 耳llustration}

Table 2 also summarizes the results for testing hypotheses about communalities based on Lawler's (1967) data. As for the methods and traits hypotheses, a series of restricted DPMs were fit to test hypotheses about communality patterns, and $\chi^{2}$ difference tests then were used to test the hypotheses.

$H_{10}$ and $H_{11}$ imply two versions of the DPM: a heteroscedastic error model and a composite error model, respectively. The latter model is a special case of the former, because $\mathrm{H}_{11}$ imposes a restriction of composite errors on $\mathbf{E}$. Table 2 shows the results for the general model (Model 
P) and for the restricted model with the composite error structure (Model Q). The $\chi^{2}$ difference test (Model $\mathrm{P}$ vs. Q) was not significant at the .99 level. Thus, $H_{11}$ could not be rejected, and the composite error model was suitable for the data.

$\mathrm{H}_{12}$ predicts that trait communalities remain the same under all methods, and it requires the restriction $\mathbb{E}_{\mathrm{M}}=\mathbb{I}$ (Model $\mathrm{R}$ in Table 2). The $\chi^{2}$ difference test (Model $Q$ vs. $R$ ) was not significant at the .20 level. Thus, $H_{12}$ could not be rejected, and there is evidence that trait communalities were constant across methods.

$\mathrm{H}_{13}$ posits that method communalities remain the same under all traits. This hypothesis was tested by examining the restriction $\mathbf{E}_{\mathrm{T}}=\mathbb{I}$ (Model S) vs. Model Q; the $\chi^{2}$ difference test was not significant at the .10 level. $\mathrm{H}_{14}$ requires that all diagonal elements of $\mathbf{E}$ are the same (Model $\mathrm{T}$ ). The $\chi^{2}$ difference test (Model Q vs. T) suggested that this hypothesis could not be rejected. This finding suggests that trait communalities remained the same under all methods and that method correlations remained the same under all traits.

\section{Conclusions}

This paper has shown how the overall and specific patterns of traits, methods, and communalities can be formally tested with the MUTMUM program. Empirical underidentification problems, which are frequently observed in MTMM analyses (Schmitt \& Stults, 1986), were not encountered in analyzing the data from Lawler (1967). These problems were avoided, perhaps because the program imposes constraints on the parameter space to avoid ill-defined solutions. In general, however, it is possible that parameters constrained at the boundaries point to misspecification errors such as when negative or zero uniqueness arises. The results also provided some useful information - the trait correlations appeared to be equal, and method and trait communalities were constant for the Lawler data. Such information could be useful in practical applications of the direct-product model.

\section{References}

Bagozzi, R. P. (1991). Further thoughts on the validity of measures of elation, gladness, and joy. Journal of Personality and Social Psychology, 61, 98-104.

Bagozzi, R. P., \& Yi, Y. (1990). Assessing method variance in multitrait-multimethod matrices: The case of self-reported affect and perceptions at work. Journal of Applied Psychology, 75, 547-560.

Bagozzi, R. P., \& Yi, Y. (1991). Multitrait-multimethod matrices in consumer research. Journal of Consumer Research, 17, 426-439.

Bagozzi, R. P., \& Yi, Y. (in press). Multitraitmultimethod matrices in consumer research: Critique and new developments. Journal of Consumer Psychology.

Bagozzi, R. P., Yi, Y., \& Phillips, L. (1991). Assessing construct validity in organizational research. $A d$ minisirative Science Quarterly, 36, 421-436.

Bentler, P. M. (1989). EQS: Structural equations program manual. Los Angeles: BMDP Statistical Software, Inc.

Bentler, P. M., Poon, W. Y., \& Lee, S. Y. (1988). Generalized multimode latent variable models: Implementation by standard programs. Computational Statistics and Data Analysis, 6, 107-118.

Browne, M. W. (1984). The decomposition of multitrait-multimethod matrices. British Journal of Mathematical and Statistical Psychology, 37, 1-21.

Browne, M. W. (1989). Relationships between an additive model and a multiplicative model for multitrait-multimethod matrices. In R. Coppi \& S. Bolasco (Eds.), Multiway data analysis (pp. 507-520). Amsterdam: North-Holland.

Browne, M. W. (1991). MUTMUM: PC user's guide [Computer program and manual]. Columbus: Ohio State University, Department of Psychology.

Campbell, D. T., \& O'Connell, E. J. (1967). Methods factors in multitrait-multimethod matrices: Multiplicative rather than additive? Multivariate $\mathrm{Be}$ havioral Research, 2, 409-426.

Campbell, D. T., \& O'Connell, E. J. (1982). Methods as diluting trait relationships rather than adding irrelevant systematic variance. In D. Brinberg \& $\mathrm{L}$. Kidder (Eds.), Forms of validity in research: New directions for methodology of social and behavioral science (Vol. 12, pp. 93-111). San Francisco: Jossey-Bass.

Cudeck, R. (1988). Multiplicative models and MTMM matrices. Journal of Educational Statistics, 13, 131-147.

Jöreskog, K. G., \& Sörbom, D. (1989). LISREL 7: A guide to the program and applications (2nd ed.) [Computer program and manual]. Chicago: SPSS, Inc. 
Lastovicka, J. L., Murry, J. P., Jr., \& Joachimsthaler, E. (1990). Evaluating the measurement validity of lifestyle typologies with qualitative measures and multiplicative factoring. Journal of Marketing Research, 28, 11-23.

Lawler, E. E., III. (1967). The multitrait-multimethod approach to measuring managerial job performance. Journal of Applied Psychology, 51, 369-381.

Schmitt, N., \& Stults, D. N. (1986). Methodology review: Analysis of multitrait-multimethod matrices. Applied Psychological Measurement, 10, $1-22$.

Swain, A. J. (1975). Analysis of parametric structures for variance matrices. Unpublished doctoral dissertation, University of Adelaide, South Africa.

Widaman, K. F. (1985). Hierarchically nested covariance structure models for multitrait-multimethod data. Applied Psychological Measurement, 9, 1-26.
Wothke, W., \& Browne, M. W. (1990). The direct product model for the MTMM matrix parameterized as a second order factor analysis model. Psychometri$k a, 55,255-262$.

\section{Acknowledgments}

The authors thank the editor and two anonymous reviewers for helpful suggestions on an earlier version of this article. An anonymous reviewer suggested testing hypotheses for communalities. The financial support of the Michigan Business School is also gratefully acknowledged.

\section{Author's Address}

Send requests for reprints or further information to Richard P. Bagozzi or Youjae Yi, The University of Michigan, School of Business Administration, Ann Arbor MI 48109-1234, U.S.A. 